\title{
Classificação de Falhas em Processos Industriais Utilizando o Algoritmo Auto-Cloud
}

\author{
Mailson Ribeiro Santos* Clauber Gomes Bezerra** \\ Luiz Affonso Guedes ${ }^{* * *}$ Yuri Thomas Pinheiro Nunes ${ }^{* * * *}$ \\ * Departamento de Engenharia de Computação e Automação, \\ Universidade Federal do Rio Grande do Norte, RN, (e-mail: \\ mayllsonribeiro6@gmail.com). \\ ** Campus Natal Zona Leste, Instituto Federal de Educação, Ciência e \\ Tecnologia do Rio Grande do Norte, RN (e-mail: \\ clauber.bezerra@ifrn.edu.br) \\ *** Departamento de Engenharia de Computação e Automação, \\ Universidade Federal do Rio Grande do Norte, RN,(e-mail: \\ affonso@dca.ufrn.br) \\ **** Departamento de Engenharia de Computação e Automação, \\ Universidade Federal do Rio Grande do Norte, RN,(e-mail: \\ yuri.tpinheiro@gmail.com)
}

\begin{abstract}
An industrial failure that is not detected in a timely manner can generate a set of undesirable consequences for humans, the environment, equipment life, and industry production. Thus, there is a high demand for secure system solutions that can identify real-time industry failures. Therefore, this work seeks to perform the industries failures classification by means of the method called Auto-Cloud that is suitable to treat data set in data stream format. For the validation of the industrial data classifier, the Tennessee Eastman benchmark was used.The Auto-Cloud was implemented in Python programming language, to perform tests it was necessary to select some flaws and process variables, from this data we generated graphs in which the algorithm identified the industrial faults, and finally they are presented contributions and ideas for future work.

Resumo: Uma falha industrial não detectada em tempo hábil pode gerar um conjunto de consequências indesejáveis aos seres humanos, ao meio ambiente, a vida útil dos equipamentos e a produção da indústria. Dessa forma, existe uma alta demanda por soluções de sistemas seguros que consigam identificar falhas indústrias em tempo real. Em vista disso, este trabalho busca realizar a classificação de falhas indústrias por meio do método denominado Auto-Cloud que é adequado para tratar conjunto de dados em formato de streams de dados. Para validação do classificador foi utilizado o benchmark Tennessee Eastman. O Auto Cloud foi implementado em linguagem de programação Python, para realização de testes foi necessário selecionar algumas falhas e variáveis de processo, a partir destes dados geramos gráficos nos quais o algoritmo identificou as falhas industriais, e, por fim são apresentadas as contribuições e ideias para trabalhos futuros.
\end{abstract}

Keywords: Data stream; Classification; Auto-Cloud; Tennessee Eastman; Evolving Systems. Palavras-chaves: Stream de dados; Classificação; Auto-Cloud;Tennessee Eastman; Sistemas Evolutivos.

\section{INTRODUÇÃO}

Hoje em dia, existem uma série de aplicações que lidam com grandes quantidades de dados. Em algumas delas, esses dados são obtidos na forma de um stream de dados. Paiva (2014) menciona que as aplicações de detecção de intrusos em redes de computadores, fluxo de transações financeiras, previsões de consumo de energia, monitora-

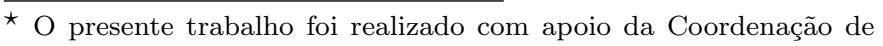
Aperfeiçoamento de Pessoal de Nível Superior - Brasil (CAPES) Código de Financiamento 001
}

mento de usuários por meio do celular e mineração de redes sociais lidam com streams de dados. De uma forma geral, a automação está em quase todos os domínios do dia a dia e as suas transações são registradas em alta velocidade, gerando grandes quantidades de dados, que podem ser analisados como streams de dados. É essencial realizar a análise destes registros para melhorar o desenvolvimento de negócios (Kokate et al., 2018).

Tarefas importantes na mineração de stream de dados são estruturar e organizar grandes quantidades de exemplos. Todavia, essas tarefas ainda são desafios, já que uma 
stream de dados possui teoricamente comprimento infinito e natureza evolutiva. Como os exemplos de uma stream de dados chegam continuamente, eles devem ser analisados uma única vez com memória e tempo limitados (Shao et al., 2018).

Os dados referentes a ocorrência de falhas em processos industriais são streams de dados. Assim, em aplicações dessa natureza, é necessário que sejam utilizados métodos que consigam tratar todas as peculiaridades deste formato de dados. Germano et al. (2016) afirma que uma falha industrial não detectada em tempo adequado pode gerar um conjunto de ações indesejáveis como paradas inesperadas, redução da vida útil do equipamento, acidentes ao meio ambiente e a vida do ser humano.

Segundo Bezerra (2017), grande parte das abordagens tradicionais propostas na literatura para tratamento de dados usam conjunto de dados offline. Já Jain et al. (1999), relata que a maioria das técnicas de aprendizagem de máquina necessitam de algum conhecimento precedente do conjunto analisado. Conforme Paiva (2014), nos últimos anos as pesquisas e processos práticos de aprendizagem de máquina concentraram-se em aplicações batch, ou seja, aplicações que possuem o conjunto de treinamento previamente disponível para processamento. Logo, a maioria das abordagens de aprendizagem de máquina disponíveis não são adequados para tratar um grande conjunto de problemas, em que os dados são apresentados como um stream.

Dentre as contribuições deste trabalho, podemos citar a realização da classificação de falhas industriais de forma online, por meio de um método evolutivo e adaptativo que trata streams de dados. Esse método foi desenvolvido por Bezerra (2017) e é denominado Auto-Cloud.

O restante deste artigo está organizado da seguinte forma: na Seção 2 são abordados os conceitos de excentricidade e tipicidade, além da descrição dos algoritmos TEDA (Typicality and Eccentricity Data Analytics) e Auto-Cloud. Na seção seguinte, Seção 3, é descrito o benchmark utilizado e os resultados obtidos na análise desse benchmark utilizando o Auto-Cloud. Por fim, a conclusão deste trabalho é feita na Seção 4 .

\section{ALGORITMO AUTO-CLOUD}

De acordo com Angelov (2014), a tipicidade refere-se a medida de quanto uma amostra é similar as demais amostras de determinado conjunto, sendo essa similaridade normalmente calculada com base em uma distância. Na Figura 1, podemos observar que o ponto A possui uma alta tipicidade em relação aos demais pontos do conjunto, já o ponto B possui uma baixa tipicidade em relação aos outros elementos. A excentricidade possui um significado antagônico em relação à tipicidade, ou seja, é a medida de quanto uma amostra difere de determinado conjunto. $\mathrm{Na}$ Figura 1, podemos observar que o ponto B possui uma alta excentricidade em relação aos demais pontos do conjunto, já o ponto A possui uma baixa excentricidade em relação aos outros elementos.

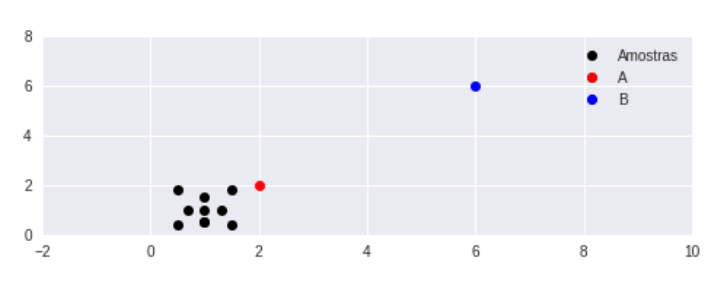

Figura 1. Exemplo de tipicidade e excentricidade

\subsection{TEDA}

O TEDA (Typicality and Eccentricity Data Analytics) é um algoritmo estatístico proposto por Angelov (2014) utilizado na detecção de outliers. O TEDA utiliza os conceitos de tipicidade e excentricidade para definir se determinado dado é "normal" (inlier) ou outlier, ou seja, uma amostra que apresenta alta excentricidade, e consequentemente baixa tipicidade, possivelmente é um outlier.

A excentricidade é definida por meio da seguinte equação recursiva (Angelov, 2014):

$$
\xi_{k}(x)=\frac{1}{k}+\frac{\left(\mu_{k}^{x}-x_{k}\right)^{T}\left(\mu_{k}^{x}-x_{k}\right)}{k\left[\sigma^{2}\right]_{k}^{x}},\left[\sigma^{2}\right]_{k}^{x}>0
$$

Onde $\xi_{k}(x)$ é a excentricidade da amostra $k$ em relação as amostras anteriores, $\left[\sigma^{2}\right]_{k}^{x}$ é a variância de $k$ amostras de $x$ e $\mu_{k}^{x}$ é a média de $k$ amostras de $x$. A partir da equação 1 , podemos verificar que para calcular a excentricidade da amostra $k$, precisamos apenas de três parâmetros $k, \mu_{k}^{x}$ e $\left[\sigma^{2}\right]_{x}^{k}$. A seguir, temos as equações para cálculo destes parâmetros de forma recursiva:

$$
\begin{gathered}
k=k+1 \\
\mu_{k}^{x}=\frac{(k-1)}{k} \mu_{k-1}^{x}+\frac{1}{k} x_{k}, \quad k \geq 1, \quad \mu_{0}^{x}=0 \\
{\left[\sigma^{2}\right]_{k}^{x}=\frac{(k-1)}{k}\left[\sigma^{2}\right]_{k-1}^{x}+\frac{1}{k}\left\|x_{k}-\mu_{k}\right\|^{2}, \quad\left[\sigma^{2}\right]_{0}^{x}=0}
\end{gathered}
$$

A tipicidade, $\tau$, da amostra $k$ de $x$ é definida como o complemento da excentricidade, a excentricidade e tipicidade normalizadas são definidas pelas respectivas equações Angelov (2014):

$$
\begin{aligned}
& \zeta_{k}(x)=\frac{\xi_{k}(x)}{2}, \sum_{i=1}^{k} \zeta_{i}(x)=1, k \geq 2 \\
& t_{k}(x)=\frac{\tau_{k}(x)}{k-2}, \sum_{i=1}^{k} t_{i}(x)=1, \quad k \geq 2
\end{aligned}
$$

O TEDA utiliza a inequação 7 para determinar se um determinado ponto é um outlier ou inlier:

$$
\zeta_{k}>\frac{m^{2}+1}{2 k}, m>0
$$

Onde $\zeta_{k}$ é a excentricidade normalizada, $k$ é a quantidade de amostras no instante atual e $m$ é a quantidade de desvios padrão utilizado. O valor do $m$ controla a sensibilidade do método. Caso o valor de $m$ seja pequeno, serão identificados muitos outliers e a medida que aumentamos esse valor, a sensibilidade do algoritmo diminui, ou seja, menos outliers serão identificados. 


\subsection{Auto-Cloud}

Auto-Cloud é um método que realiza classificação e agrupamento de dados no formato de streams de dado é baseado no algoritmo TEDA e utiliza estruturas denominadas data clouds para agrupar os dados (Bezerra, 2017).

Data clouds são estruturas utilizadas para agrupar um conjunto de dados que não possuem formato pré-definido. Uma data cloud representa um conjunto de dados com características semelhantes. Já diferentes data clouds representam conjuntos com baixa similaridade entre si Angelov (2014). Na Figura 2 temos duas data cloud $c 1$ e $c 2$, sendo que $c 1$ está ligado com os dados por linhas tracejadas e $c 2$ por linhas pontilhadas.

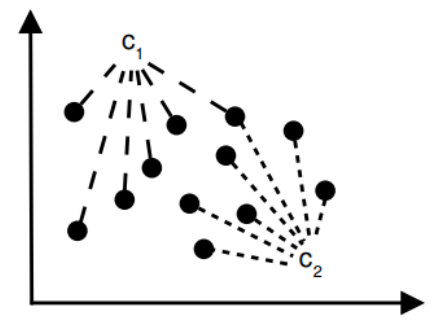

Figura 2. Exemplos de data cloud. FONTE: Bezerra (2017)

As data clouds apresenta uma representação mais fiel do conjunto de dados analisado, visto que não possuem limites geométricos. O algoritmo Auto-Cloud define para cada amostra um grau de pertinência fuzzy em relação a cada data cloud existente. Assim, determinada amostra pode pertencer simultaneamente a todas as data clouds com diferentes graus de pertinência, $\gamma \in[0 ; 1]$. O grau de pertinência é obtido por meio do cálculo da tipicidade normalizada, definida na equação 6 (Bezerra, 2017). Na Figura 2 podemos ver uma amostra que pertence simultaneamente às duas data clouds $c 1$ e $c 2$. Como relatado anteriormente, o Auto-Cloud é baseado no TEDA, sendo que as equações do Auto-Cloud podem ser vistas como uma generalização das equações do TEDA para cada data cloud.

O Auto-Cloud necessita armazenar três informações para cada data cloud $c_{i}$ :

- $s_{k}^{i}$ : o número de amostras da data cloud $i$ após ler $\mathrm{k}$ amostras.

- $\mu_{k}^{i}$ : a média amostral da data cloud $i$ após ler $\mathrm{k}$ amostras.

- $\left[\sigma^{2}\right]_{k}^{i}$ : a variância amostral da data cloud $i$ após ler $\mathrm{k}$ amostras.

\subsection{Atualização das data clouds}

O Auto-Cloud determina se uma amostra pertence a determinada data cloud $c_{i}$ por meio da excentricidade normalizada $\zeta_{i}\left(x_{k}\right)$ da amostra $x_{k}$, conforme ilustrado na Figura 3. Se a excentricidade tiver um valor alto, o algoritmo considera que a amostra possui características significativamente diferentes das amostras presentes na data cloud $c_{i}$, logo não é realizada nenhuma alteração na data cloud $c_{i}$. Se a excentricidade tiver um valor baixo o algoritmo considera que a nova amostra possui alta similaridade com a data cloud $c_{i}$, portanto, são atualizados os valores das três características armazenadas na data cloud $c_{i}$. Se uma amostra analisada não pertencer a nenhuma data cloud existente é criada uma nova para armazenar está amostra.

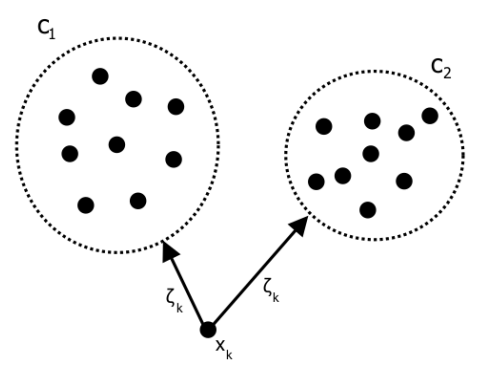

Figura 3. Funcionamento do algoritmo Auto-Cloud. FONTE: Bezerra (2017)

A seguir temos as equações utilizadas para calcular a excentricidade, $\xi^{i}\left(x_{k}\right)$, e a excentricidade normalizada, $\zeta^{i}\left(x_{k}\right)$, da amostra, $x_{k}$, em relação a $i$-ésima data cloud Bezerra (2017):

$$
\begin{gathered}
\xi^{i}\left(x_{k}\right)=\frac{1}{\left[s_{k}^{i}\right]^{\prime}}+\frac{\left(\left[\mu_{k}^{i}\right]^{\prime}-x_{k}\right)^{T}\left(\left[\mu_{k}^{i}\right]^{\prime}-x_{k}\right)}{\left[s_{k}^{i}\right]^{\prime}\left[\left[\sigma^{2}\right]_{k}^{i}\right]^{\prime}} \\
\zeta^{i}\left(x_{k}\right)=\frac{\xi^{i}\left(x_{k}\right)}{2}
\end{gathered}
$$

Onde $\left[s_{k}^{i}\right]^{\prime}$ é o valor temporário do número de amostras da $i$-ésima data cloud $c_{i},\left[\mu_{k}^{i}\right]^{\prime}$ é o valor temporário da média das amostras da i-ésima data cloud $c_{i}$ e $\left[\left[\sigma^{2}\right]_{k}^{i}\right]^{\prime}$ é o valor temporário da variância das amostras da $i$-ésima data cloud $c_{i}$. A seguir temos as equações utilizadas para calcular estes valores:

$$
\begin{gathered}
{\left[s_{k}^{i}\right]^{\prime}=s_{k-1}^{i}+1} \\
{\left[\mu_{k}^{i}\right]^{\prime}=\frac{\left[s_{k}^{i}\right]^{\prime}-1}{\left[s_{k}^{i}\right]^{\prime}} \mu_{k-1}^{i}+\frac{1}{\left[s_{k}^{i}\right]^{\prime}} x_{k}} \\
{\left[\left[\sigma^{2}\right]_{k}^{i}\right]^{\prime}=\frac{\left[s_{k}^{i}\right]^{\prime}-1}{\left[s_{k}^{i}\right]^{\prime}}\left[\sigma^{2}\right]_{k-1}^{i}+\frac{1}{\left[s_{k}^{i}\right]^{\prime}}\left\|x_{k}-\left[\mu_{k}^{i}\right]^{\prime}\right\|}
\end{gathered}
$$

Os valores de $s_{k}^{i}, \mu_{k}^{i}$ e $\left[\sigma^{2}\right]_{k}^{i}$ só vão ser atualizados respectivamente pelos valores de $\left[s_{k}^{i}\right]^{\prime},\left[\mu_{k}^{i}\right]^{\prime}$ e $\left[\left[\sigma^{2}\right]_{k}^{i}\right]^{\prime}$ calculados pelas equações 10,11 e 12 , se a amostra, $x_{k}$, pertencer ao data cloud $c_{i}$. Para determinar se uma amostra pertence à data cloud $c_{i}$ usamos a seguinte equação:

$$
\zeta^{i}\left(x_{k}\right) \leq \frac{m^{2}+1}{2 s_{k}^{i}}
$$

Onde, $\mathrm{m}$ é a sensibilidade do algoritmo. Se a equação 13 for verdadeira, então a amostra $x_{k}$ pertence a data cloud $c_{i}$.

\subsection{Fusão das Data cloud}

O Auto-Cloud possui a capacidade de realizar a fusão de duas data clouds que possuem alta similaridade. O processo de fusão ocorre quando o número de dados que pertence simultaneamente às duas estruturas, ou seja, a intersecção das data clouds é considerado alto.

Segundo Bezerra (2017), a fusão é realizada quando uma das seguintes equações é considerada verdadeira:

$$
s_{k}^{c_{i} \cap c_{j}}>s_{k}^{i}-s_{k}^{c_{i} \cap c_{j}}
$$




$$
s_{k}^{c_{i} \cap c_{j}}>s_{k}^{j}-s_{k}^{c_{i} \cap c_{j}}
$$

Onde $s_{k}^{c_{i} \cap c_{j}}$ é a intersecção entres as data clouds $c_{i}$ e $c_{j}$. Essas condições mostram que duas data clouds passam pelo processo de fusão quando a quantidade de amostras na intersecção das data clouds é maior que a quantidade de amostras que pertence apenas a uma dessas estruturas.

Na Figura 4 podemos ver o processo de merge realizado pelo Auto-Cloud. A Figura 4(a) mostra um conjunto de dados no qual não é realizado fusão, visto que a intersecção de $c_{1}$ e $c_{2}$ é menor que o número de amostra que pertence exclusivamente a $c_{1}$ e $c_{2}$. Já na Figura 4(b) é realizada a fusão das duas data clouds, pois a intersecção de $c_{1}$ e $c_{2}$ é maior que o número de amostras que pertence exclusivamente a $c_{2}$. Na Figura 4(c) temos o resultado da fusão realizada entre $c_{1}$ e $c_{2}$ mostradas na Figura 4(b).

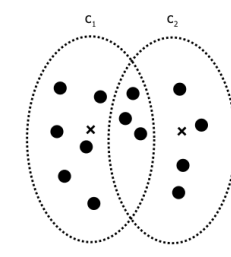

(a)

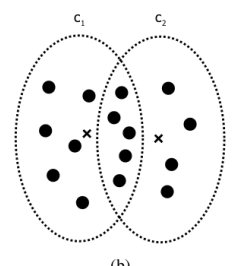

(b)

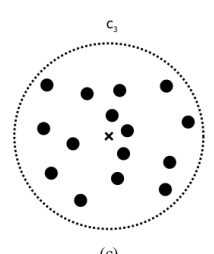

Figura 4. Fusão de duas data clouds $c_{1}$ e $c_{2}$. FONTE: Bezerra (2017)

Quando ocorre a fusão de dois data clouds, $c_{i}$ e $c_{j}$, as características são atualizadas pelas seguintes equações Bezerra (2017):

$$
\begin{gathered}
s_{k}^{l}=s_{k}^{i}+s_{k}^{j}-s_{k}^{c_{i} \cap c_{j}} \\
\mu_{k}^{l}=\frac{s_{k}^{i} \mu_{i}+s_{k}^{j} \mu_{j}}{s_{k}^{i}+s_{k}^{j}} \\
{\left[\sigma^{2}\right]_{k}^{l}=\frac{\left(s_{k}^{i}-1\right) \sigma_{i}^{2}+\left(s_{k}^{j}-1\right) \sigma_{j}^{2}}{s_{k}^{i}+s_{k}^{j}-2}}
\end{gathered}
$$

\subsection{Classificação dos Dados}

O classificador proposto por Bezerra (2017) é baseado no conceito do sistema fuzzy AnYa Angelov and Yager (2012), que apresenta como principal vantagem em relação ao sistema fuzzy Mamdani and Assilian (1975) e TakagiSugeno a não necessidade de parâmetros, conectores lógicos, operações de agregação ou funções de pertinência nos antecedentes das regras fuzzy (Bezerra, 2017).

Para cada amostra analisada pelo Auto-Cloud é gerada uma nova saída que indica a quais classes essa amostra pertence, ou seja, para cada amostra processada é gerado um vetor de graus de pertinência para todas as data clouds existentes. O grau de pertinência de determinada amostra $x_{k}$ é dado pelo valor da tipicidade normalizada, $t_{k}^{i}$. O valor da tipicidade normalizada pode ser calculada pelas seguintes equações (Bezerra, 2017):

$$
\begin{gathered}
\xi_{k}^{i}=\frac{1}{s_{k}^{i}}+\frac{\left(\mu_{k}^{i}-x_{k}\right)^{T}\left(\mu_{k}^{i}-x_{x}\right)}{s_{k}^{i}\left[\sigma^{2}\right]_{k}^{i}} \\
\tau_{k}^{i}=1-\xi_{k}^{i}(x) \\
\zeta_{k}^{i}(x)=\frac{\xi_{k}^{i}(x)}{2}, \sum_{l=1}^{k} \xi_{l}^{i}(x)=1, k>=2
\end{gathered}
$$

$$
t_{k}^{i}(x)=\frac{\tau_{k}^{i}(x)}{k-2}, \sum_{l=1}^{k} t_{l}^{i}(x)=1, k>=2
$$

Onde $t_{k}^{i}(x)$ informa quanto uma amostra $k$ de $x$ é similar a determinada data cloud $c_{i}$. A pertinência $\alpha$, da amostra $x_{k}$ com relação a data cloud $c_{i}$ é definida pela equação 23 .

$$
\alpha_{c_{i}}\left(x_{k}\right)=\left\{\begin{array}{l}
t_{k}^{i}(x) \text { se } x \in c_{i} \\
0 \text { caso contrário }
\end{array}\right.
$$

Já a pertinência normalizada é definida pela equação 24 (Bezerra, 2017):

$$
\gamma_{c_{i}}\left(x_{k}\right)=\frac{\alpha_{c_{j}}\left(x_{k}\right)}{\sum_{j=1}^{N} \alpha_{c_{j}}\left(x_{k}\right)}
$$

Onde $\mathrm{N}$ representa o número de data cloud existente, o Auto-Cloud gera uma rotulação final de cada amostra por meio de um classificador crisp e utiliza a regra "the winner takes it all"Ishibuchi et al. (1995), a seguir temos a forma de rotulação de cada amostra:

$$
\text { Classe }=\text { Classe }^{l^{*}}, l^{*}=\operatorname{argmax}_{l=1}^{N}\left(\gamma_{c l}\left(x_{K}\right)\right)
$$

A classe a que a amostra processada pertence é definida pelo maior grau de pertinência.

O algoritmo Auto-Cloud foi implementado na linguagem de programação Python utilizando o paradigma de Programação Orientado a Objetos, para facilitar a implementação usou-se a biblioteca Numpy, que é um pacote para computação científica que fornece um Array $\mathrm{N}$-dimensional poderoso, possuir funções para tratar problemas relacionados à Álgebra Linear e possui a capacidade de manipular números aleatórios.

\section{RESULTADOS}

O Tennessee Eastman Procces (TEP) foi desenvolvido pela Eastman Chemical Company com o objetivo de disponibilizar um simulador baseado em um processo químico real para avaliar o controle de processos e métodos de monitoramento Downs and Vogel (1993). Mesmo o TEP sendo um processo antigo, ainda é uma ferramenta importante para validação de algoritmos, visto que é um modelo não-linear de um sistema de multicomponentes bastante complexo (Bathelt et al., 2015). O processo descrito pelo TEP é formado por quatro reagentes gasosos $\mathrm{A}, \mathrm{C}, \mathrm{D}$ e $\mathrm{E}$, e o componente inerte $\mathrm{B}$, um subproduto $\mathrm{F}$ e dois produtos líquidos, G e H. A seguir, temos a equação que descreve o processo TEP.

$$
\begin{array}{r}
A(g)+C(g)+D(g) \rightarrow G(l) \\
A(g)+C(g)+E(g) \rightarrow H(l) \\
A(g)+E(g) \rightarrow F(l) \\
3 D(g) \rightarrow 2 F(l)
\end{array}
$$

O processo se inicia com os reagentes gasosos no reator, posteriormente o produto do reator é resfriado por meio de um condensador e, em seguida, um separador líquido gasoso retira o produto gasoso e este é reciclado para a alimentação do reator. Parte deste produto de reciclagem deve ser purgado para evitar acúmulo do subproduto e do componente inerte. Os componentes condensados são enviados para stripper e são retirados reagentes remanescentes. 
Por fim, os produtos $\mathrm{G}$ e H são aplicados a uma seção de refinação que não está incluído na planta.

O Auto-Cloud foi implementado conforme descrito anteriormente, sendo escolhido o valor de $m$ igual a 2.5, logo depois foi necessário escolher algumas variáveis de processo da planta que manifestaram ter um conjunto maior de informações sobre a falha analisada. Essa seleção foi necessária, pois, a planta possui muitas variáveis e normalmente poucas destas variáveis são afetadas por determinada falha, utilizamos as variáveis 1 e 10 para realização dos testes que representam respectivamente a vazão de A e vazão de purga.

Ao analisar o conjunto de variáveis nos períodos de falhas foi possível observar que existe um conjunto de falhas em que as variáveis de processo não são afetadas. Dessa forma, essas falhas não são analisadas neste estudo. A seguir, temos os resultados obtidos em sinais que possuem mais de uma falha, cada sinal adquirido permanece por um período sem falha de 60 horas (6000 amostras), em seguida é adicionada uma falha por 20 horas (2000 amostras), posteriormente são adicionadas novas falhas seguindo este padrão de 60 horas sem falha e 20 horas com falha. Os diferentes períodos de falhas estão separados nos gráficos por linha verticais.

\subsection{Falha 21}

Foi selecionado $m$ igual a 2.5 para realização do teste, sendo possível observar nas Figuras 5 e 6 que o Auto-Cloud consegue identificar três classes: duas referente as falhas e uma relativo ao comportamento normal da planta.

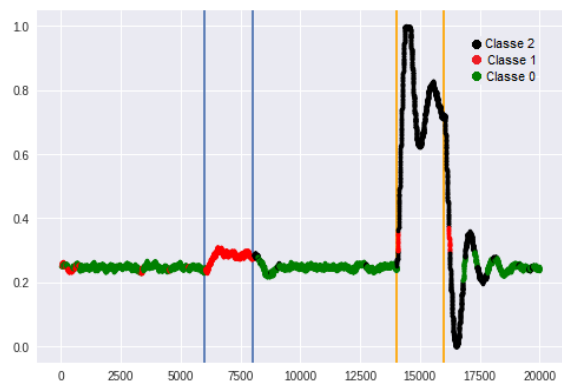

Figura 5. Falha 2 e 1 variável 1.

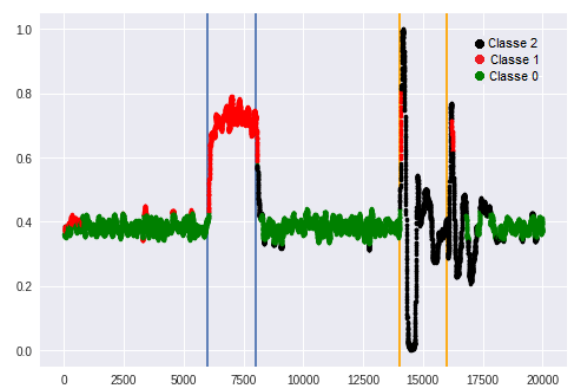

Figura 6. Falha 2 e 1 variável 10.

\subsection{Falha 26}

Inicialmente, foi definido limiar igual 2.5 gerando os resultados apresentados nas Figuras 7 e 8. Posteriormente, o limiar foi alterado para 3.5 e gerados novos resultados apresentados nas Figuras 9 e 10.

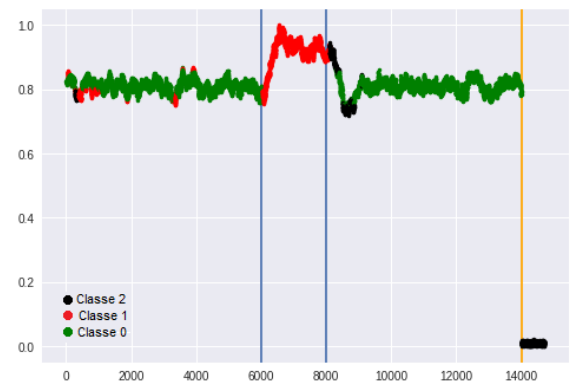

Figura 7. Falha 2 e 6 variável 1.

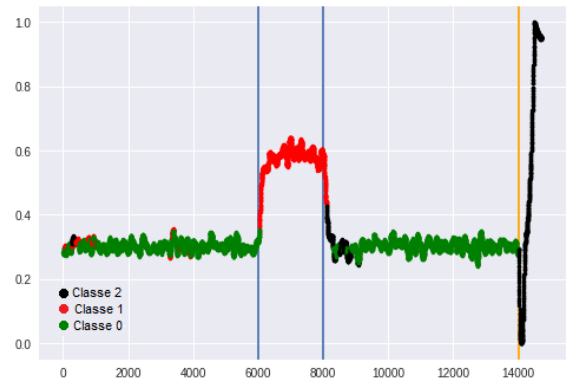

Figura 8. Falha 2 e 6 variável 10.

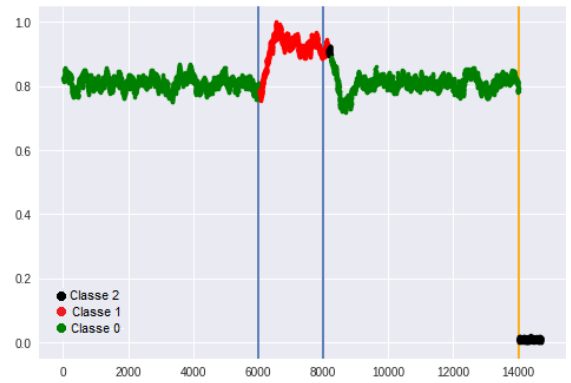

Figura 9. Falha 2 e 6 variável 1.

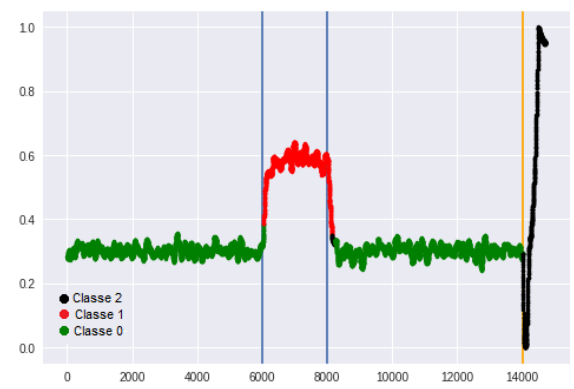

Figura 10. Falha 2 e 6 variável 10.

Os resultados apresentados anteriormente são satisfatórios, pois, o algoritmo consegue identificar as regiões com falha e sem falha para os dois limiares utilizados, assim como é possível identificar que o resultado apresentado com limiar igual 3.5 foi melhor. 


\subsection{Falha 216}

Nas Figuras 12 e 11 são apresentados os gráficos referentes às variáveis 1 e 10 no qual o Auto-Cloud encontrou 4 classes distintas, onde uma é referente a estado normal da planta e as outras três são referentes às falhas 2,1 e 6 .

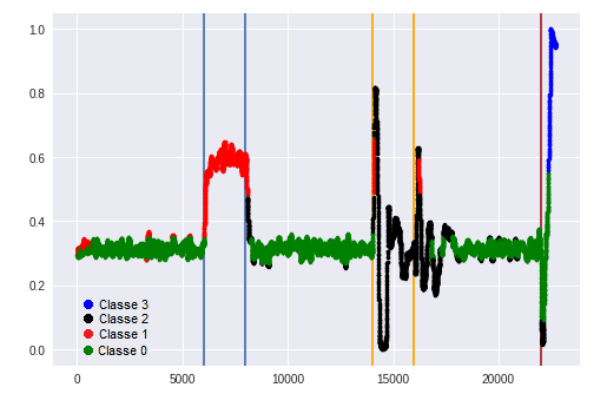

Figura 11. Falha 2, 1 e 6 variável 10.

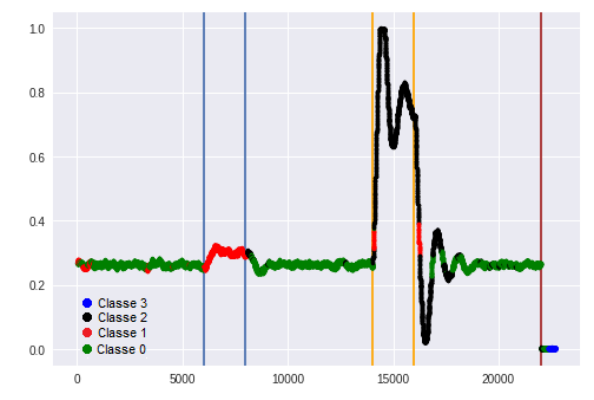

Figura 12. Falha 2, 1 e 6 variável 1.

\section{CONCLUSÕES}

O método utilizado para detecção e classificação de falhas industriais apresentou-se adequado aos processos industriais e pode ser aplicado em diversas indústrias, visto que as únicas informações necessárias para sua aplicação são as variáveis de processo e o limiar $m$ utilizado pelo algoritmo. Os resultados obtidos apresentaram satisfatórios, todavia ainda existem características no Auto-Cloud que podem ser aprimoradas.

Uma dificuldade encontrada durante o desenvolvimento deste trabalho foi a seleção das variáveis de processo, pois, é um processo manual e não garante selecionar as variáveis mais adequadas. Desta forma, um importante trabalho futuro é a realização de pesquisa sobre a seleção automática das variáveis utilizadas pelo Auto-Cloud.

Outra dificuldade encontrada durante o desenvolvimento dos experimentos foi a seleção do limiar $m$ mais adequado, logo um aprimoramento desejável seria a construção de um limiar adaptativo, que fosse atualizado a cada instante de acordo com as amostras processadas. Esse limiar adaptativo pode ser obtido por meio de uma medida de dispersão de dados, por exemplo, a medida curtose que é uma medida que caracteriza o achatamento da curva de distribuição de dados. Portanto, a implementação deste limiar adaptativo ótimo pode gerar resultados bastante satisfatórios visto a melhora apresentada provocada pela alteração deste limiar na classificação das falhas 2 e 6 .
Durante o desenvolvimento do cálculo da excentricidade foi utilizada como métrica de similaridade a distância euclidiana, todavia em algumas situações essa métrica pode não ser a medida mais adequada. Nessas situações, podemos aplicar a distância mahalanobis, pois, gera a correlação entre as amostras, sendo assim invariante as escalas.

\section{REFERÊNCIAS}

Angelov, P. (2014). Anomaly detection based on eccentricity analysis. IEEE Symposium Series in Computational Intelligence (SSCI 2014).

Angelov, P. and Yager, R. (2012). A new type of simplified fuzzy rule-based system. International Journal of General Systems.

Bathelt, A., Ricker, N.L., and Jelali, M. (2015). Revision of the tennessee eastman process model. IFACPapersOnLine.

Bezerra, C.G. (2017). Uma Abordagem Baseada em Tipicidade e Excentricidade para Agrupamento e Classificação de Streams de Dados. Ph.D. thesis, UFRN, Natal, RN.

Downs, J.J. and Vogel, E.F. (1993). A plant-wide industrial process control problem. Computers \& Chemical Engineering.

Germano, A.L., Guedes, L.A., Costa, B.S.J., and Bezerra, C.G. (2016). DetecÇÃ̃o de falhas no processo tennessee eastman utilizando $\sim$ metricas de tipicidade e excentricidade.

Ishibuchi, H., Nozaki, K., Yamamoto, N., and Tanaka, H. (1995). Selecting fuzzy if-then rules for classification problems using genetic algorithms. IEEE TRANSACTIONS ON FUZZY SYSTEMS.

Jain, A.K., Murty, M.N., and Flynn, P.J. (1999). Data clustering: A review. ACM Comput.

Kokate, U., Deshpande, A., Mahalle, P., and Patil, P. (2018). Data stream clustering techniques, applications, and models: Comparative analysis and discussion. Big Data and Cognitive Computing, 2(4). doi:10.3390/ bdcc2040032. URL http: //www . mdpi . com/2504-2289/ 2/4/32.

Mamdani, E.H. and Assilian, S. (1975). An experiment in linguistic synthesis with a fuzzy logic controller. International Journal of Man-Machine Studies.

Paiva, E.R.F. (2014). Detecção de novidade em Fluxos continuos de dados multiclasse. Ph.D. thesis, USP, São Carlos, SP.

Shao, J., Tan, Y., Gao, L., Yang, Q., Plant, C., and Assent, I. (2018). Synchronization-based clustering on evolving data stream. Information Sciences. doi:10.1016/j.ins. 2018.09.035. 